SCALAR POTENTIAL FOR CHARGE DISTRIBUTIONS WITH ELLIPSOIDAL SYMMETRY

R. L. Gluckstern*

May 20,1986

* Physics Department, University of Maryland, College Park, Maryland 20742 


\title{
SCALAR POTENTIAL FOR CHARGE DISTRIBUTIONS WITH ELLIPSOIDAL SYMMETRY
}

\author{
R. L. Gluckstern*
}

May 20, 1986

\section{Introduction}

The calculation of the electrostatic potential due to a charge distribution with "ellipsoidal symmetry". is most easily carried out in ellipsoidal coordinates. Since these may be unfamiliar to some readers, the present note outlines the calculation in some detail.

\section{Ellipsoidal Shell}

Let us assume a charge density which is a function of the variable

$$
s=\left(x^{2} / a^{2}\right)+\left(y^{2} / b^{2}\right)+\left(z^{2} / c^{2}\right)
$$

and has the form

$$
p(x, y, z)=f\left(\frac{x^{2}}{a^{2}}+\frac{y^{2}}{b^{2}}+\frac{z^{2}}{c^{2}}\right)=\int d s f(s) \delta\left(s-\frac{x^{2}}{a^{2}}-\frac{y^{2}}{b^{2}}-\frac{z^{2}}{c^{2}}\right) .
$$

We shall first explore the potential and field due to a shell of charge whose density is

$$
2 \delta\left(s-\frac{x^{2}}{a^{2}}-\frac{y^{2}}{b^{2}}-\frac{z^{2}}{c^{2}}\right) \text {. }
$$

The surface charge density on this ellipsoidal shell is not uniform. It is governed by the coordinate $w$, normal to the surface, whose direction cosines $\ell, m, n$, are determined by

$$
\mathrm{ds} / 2=\frac{\mathrm{x}}{\mathrm{a}^{2}} \mathrm{dx}+\frac{\mathrm{y}}{\mathrm{b}^{2}} \mathrm{dy}+\frac{\mathrm{z}}{\mathrm{c}^{2}} \mathrm{dz}=\frac{\ell d x+m d y+n d z}{\sigma(x, y, z)},
$$

\footnotetext{
* Physics Department, University of Maryland, College Park, MD, 20742
} 
where

$$
\sigma(x, y, z)=\left(x^{2} / a^{4}+y^{2} / b^{4}+z^{2} / c^{4}\right)^{-\frac{1}{2}}
$$

and

$$
\ell=\left(x / a^{2}\right) \sigma, \quad m=\left(y / b^{2}\right) \sigma, \quad n=\left(z / c^{2}\right) \sigma .
$$

Since the coordinate perpendicular to the surface satisfies

$$
d w=\ell d x+m d y+n d z,
$$

we can write the charge density as

$$
2 \delta\left(s-\frac{x^{2}}{a^{2}}-\frac{y^{2}}{b^{2}}-\frac{z^{2}}{c^{2}}\right)=\sigma \delta(w)
$$

corresponding to a surface charge density $\sigma$.

III. Field Inside the Ellipsoidal Shell

Consider the point $P$ within the shell and a small conical spherical angle $d \Omega$ which intersects areas $d A_{1}$ and $\mathrm{dA}_{2}$ on the surface of the shell. The "radial" vectors $\left(\vec{r}_{1}, \vec{r}_{2}\right)$ have the direction cosines

$$
\pm\left(l_{0}, m_{0}, n_{0}\right)= \pm\left(\frac{x_{1}-x_{2}}{r_{12}}, \frac{y_{1}-y_{2}}{r_{12}}, \frac{z_{1}-z_{2}}{r_{12}}\right)
$$

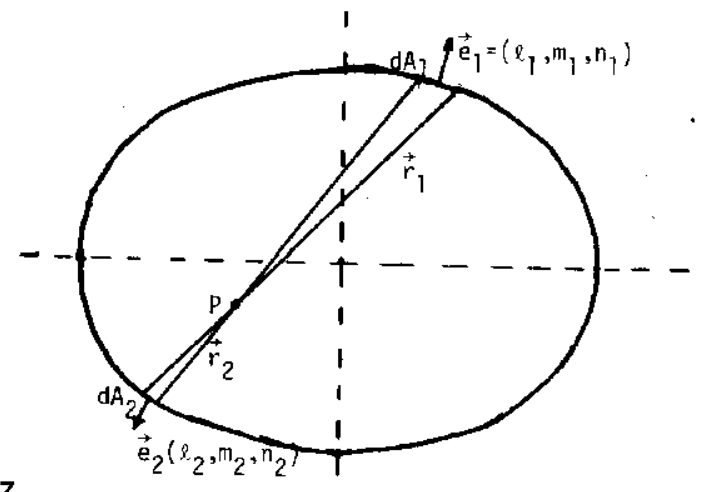

The solid angles are defined by $d \Omega=d \Omega_{1}=d \Omega_{2}$ with

$$
d \Omega_{1}=\frac{d A_{1}}{r_{1}^{2}}\left(\vec{e}_{1} \cdot \vec{e}_{0}\right), \quad d \Omega_{2}=\frac{-d A_{2}}{r_{2}^{2}}\left(\vec{e}_{2} \cdot \vec{e}_{0}\right)
$$

where the unit vectors $\vec{e}_{0}, \vec{e}_{1}, \vec{e}_{2}$ have components $\left(\ell_{0}, m_{0}, n_{0}\right),\left(\ell_{1}, m_{1}, n_{1}\right)$, $\left(l_{2}, m_{2}, n_{2}\right)$.

The contribution to the field at $P$ due to $d A_{1}$ and $d A_{2}$, with surface charge densities $\sigma_{1}$ and $\sigma_{2}$ is 


$$
d \vec{E}_{p}=\vec{e}_{0}\left(\frac{\sigma_{1} d A_{1}}{r_{1}^{2}}-\frac{\sigma_{2} d A_{2}}{r_{2}^{2}}\right)=\vec{e}_{0} d \Omega\left(\frac{\sigma_{1}}{\vec{e}_{1} \cdot \vec{e}_{0}}+\frac{\sigma_{2}}{\vec{e}_{2} \cdot \vec{e}_{0}}\right)
$$

Using (2.6) and (3.1), we have

$$
\begin{aligned}
& r_{12} \vec{e}_{0} \cdot\left(\frac{\vec{e}_{1}}{\sigma_{1}}+\frac{\vec{e}_{2}}{\sigma_{2}}\right) \\
= & \left(x_{1}-x_{2}\right)\left(x_{1}+x_{2}\right) / a^{2}+\left(y_{1}-y_{2}\right)\left(y_{1}+y_{2}\right) / b^{2}+\left(z_{1}-z_{2}\right)\left(z_{1}+z_{2}\right) / c^{2} \\
= & \left(x_{1}^{2} / a^{2}+y_{1}^{2} / b^{2}+z_{1}^{2} / c^{2}\right)-\left(x_{2}^{2} / a^{2}+y_{2}^{2} / b^{2}+z_{2}^{2} / c^{2}\right)=0 .
\end{aligned}
$$

Thus

$$
\frac{\overrightarrow{\mathrm{e}}_{1} \cdot \overrightarrow{\mathrm{e}}_{0}}{\sigma_{1}}=-\frac{\overrightarrow{\mathrm{e}}_{2} \cdot \overrightarrow{\mathrm{e}}_{0}}{\sigma_{2}}
$$

and

$$
\overrightarrow{d E}_{p}=0
$$

We have therefore shown that the field inside the ellipsoidal shell defined in (2.3) vanishes, due to pair cancellation, as occurs in the uniform spherica? charge shell.

\section{Ellipsoidal Coordinates}

Using Stratton's ${ }^{2}$ notation with a scale change for $x, y, z$, the ellipsoidal coordinates are defined for $a>b>c$ by

$$
\begin{array}{ll}
\frac{x^{2}}{a^{2}+\xi}+\frac{y^{2}}{b^{2}+\xi}+\frac{z^{2}}{c^{2}+\xi}=s, & -c^{2}<\xi \\
\frac{x^{2}}{a^{2}+n}+\frac{y^{2}}{b^{2}+n}-\frac{z^{2}}{-c^{2}-n}=s, & -b^{2}<n<-c^{2} \\
\frac{x^{2}}{a^{2}+\zeta}-\frac{y^{2}}{-b^{2}-\zeta}-\frac{z^{2}}{-c^{2}-\zeta}=s, & -a^{2}<\zeta<-b^{2}
\end{array}
$$


The coordinates $\eta, \zeta$ correspond to angle-like coordinates, while $\xi$ corresponds to a radial-1ike coordinate. (In fact, $\xi \rightarrow r^{2} / s$ as $r \rightarrow \infty$.)

The Laplacian separates in such a way that the solutions inside and outside the shell can be written as product functions in each of the variables $\xi, \eta, \zeta$. Since we already know that the solution inside the shell is constant, and since the region inside and outside the shel1 is defined by $\xi<0$ and $\xi>0$, continuity of the potential at the shel1 requires independence of $\eta$ and $\zeta$ both inside and outside the shel1. The potential $\phi$ is therefore a function only of $\xi$, and Laplace's equation can be written as

$$
\nabla^{2} \phi(\xi)=\frac{4}{s(\xi-\eta)(\xi-\zeta)} R(\xi) \frac{d}{d \xi}\left(R(\xi) \frac{d \phi}{d \xi}\right)
$$

where

$$
R(t)=\left\{\left(t+a^{2}\right)\left(t+b^{2}\right)\left(t+c^{2}\right)\right\}^{\frac{1}{2}}
$$

Thus

$$
\phi(\xi)=B \int_{\xi}^{\infty} \frac{d t}{R(t)}, \quad \xi>0
$$

is the solution of the potential outside the shell, and

$$
\phi(\xi)=B \int_{0}^{\infty} \frac{d t}{R(t)}, \quad-c^{2}<\xi<0
$$

is the solution inside the shell. Clearly $\nabla^{2} \phi(\xi)$ vanishes for $\xi>0, \xi<0$. Near $\xi=0$, the discontinuity in the slope of $\mathrm{R}(\xi) \mathrm{d} \phi / \mathrm{d} \xi$ leads to

$$
\nabla^{2} \phi(\xi)=\frac{-4 \mathrm{~B}}{s(\xi-n)(\xi-\zeta)} R(\xi) \delta(\xi)=-\frac{4 \mathrm{~B} a b c}{s n \zeta} \delta(\xi) .
$$

If we differentiate (4.1) with respect to $x, y, z, \xi$, we obtain

$$
2\left(\frac{x d x}{a^{2}+\xi}+\frac{y d y}{b^{2}+\xi}+\frac{z d z}{c^{2}+\xi}\right)=\left(\frac{x^{2}}{\left(a^{2}+\xi\right)^{2}}+\frac{y^{2}}{\left(b^{2}+\xi\right)^{2}}+\frac{z^{2}}{\left(c^{2}+\xi\right)^{2}}\right) d \xi \text {. }
$$

Near $\xi=0$, this can be written as 
- 5 -

$$
\mathrm{ds}=\mathrm{d} \xi / \sigma^{2}
$$

Using

$$
\begin{aligned}
& x^{2} / s=\frac{\left(\xi+a^{2}\right)\left(\eta+a^{2}\right)\left(\zeta+a^{2}\right)}{\left(a^{2}-b^{2}\right)\left(a^{2}-c^{2}\right)} \\
& y^{2} / s=\frac{\left(\xi+b^{2}\right)\left(n+b^{2}\right)\left(-\zeta-b^{2}\right)}{\left(a^{2}-b^{2}\right)\left(b^{2}-c^{2}\right)} \\
& z^{2} / s=\frac{\left(\xi+c^{2}\right)\left(-n-c^{2}\right)\left(-\zeta-c^{2}\right)}{\left(a^{2}-c^{2}\right)\left(b^{2}-c^{2}\right)},
\end{aligned}
$$

one can show, for $\xi=0$, that

$$
\frac{1}{\sigma^{2}}=x^{2} / a^{4}+y^{2} / b^{4}+z^{2} / c^{4}=\frac{s n \zeta}{a^{2} b^{2} c^{2}}
$$

Thus

$$
d s=\frac{s n \zeta}{a^{2} b^{2} c^{2}} d \xi
$$

and (4.8) becomes

$$
\nabla^{2} \phi(\xi)=-\frac{4 B a b c}{s n \zeta} \delta(\xi)=-\frac{4 B}{a b c} \delta\left(s-\frac{x^{2}}{a^{2}}-\frac{y^{2}}{b^{2}}-\frac{z^{2}}{c^{2}}\right)
$$

confirming the uniform density within each shell. Comparison with (2.2) then leads to

$$
B=\frac{a b c}{4 \varepsilon_{0}} f(s)
$$

and

$$
\phi(x, y, z)=\frac{a b c}{4 \varepsilon_{0}} \int_{0}^{\infty} d s f(s)\left\{\begin{array}{ll}
f^{\infty} \frac{d t}{R(s)}, & \xi>0 \\
\int_{0}^{\infty} \frac{d t}{R(t)}, & \xi<0
\end{array}\right\}
$$


The region of integration is shown shaded in the figure.

$$
\frac{x^{2}}{a^{2}+t}+\frac{y^{2}}{b^{2}+t}+\frac{z^{2}}{c^{2}+t}=s(t)
$$

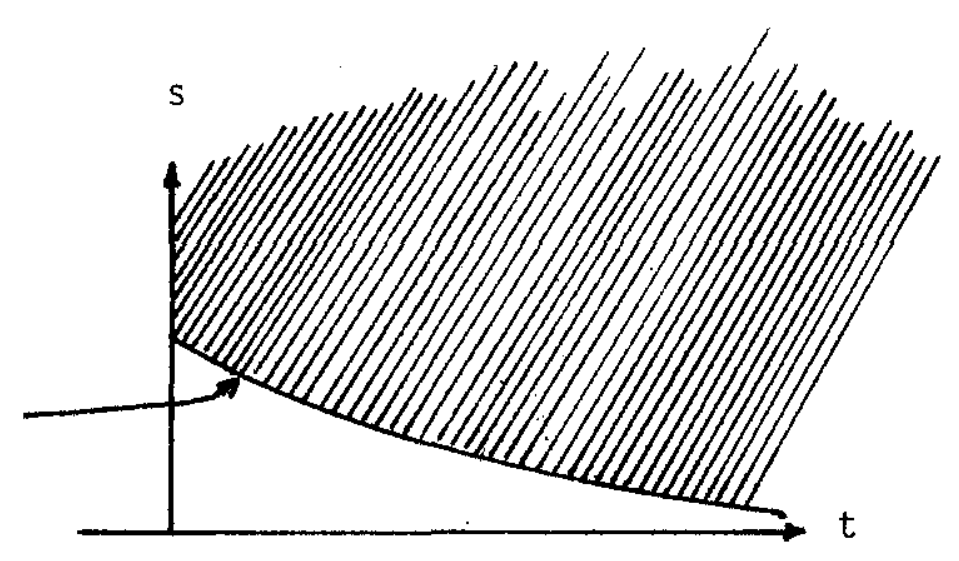

Interchanging the order of integration leads to

$$
\phi(x, y, z)=\frac{a b c}{4 \varepsilon_{0}} \int_{0}^{\infty} \frac{d t}{R(t)} \int_{s(t)}^{\infty} d s f(s)
$$

or

$$
\phi(x, y, z)=-\frac{a b c}{4 \varepsilon_{0}} \int_{0}^{\infty} \frac{d t}{R(t)} \int_{0}^{s(t)} d s f(s)
$$

where (4.19) is normalized such that $\phi(\infty)=0$, and where (4.20) is normalized such that $\phi(0)=0$.

If the functional form of the charge density, $f(s)$, is known and can be integrated, $(4.19)$ and $(4.20)$ reduce to a single integral.

V. Special Cases

A. 3-D Gaussian charge distribution

If we choose a Gaussian charge distribution defined by

$$
f(s)=\frac{Q}{\pi^{3 / 2} a b c} e^{-s},
$$

where $Q$ is the total charge, one finds from (4.19) 


$$
\phi_{3 G}(x, y, z)=\frac{Q}{4 \pi^{3 / 2} \varepsilon_{0}} \int_{0}^{\infty} \frac{\exp \left(-\frac{x^{2}}{a^{2}+t}-\frac{y^{2}}{b^{2}+t}-\frac{z^{2}}{c^{2}+t}\right)}{\left\{\left(a^{2}+t\right)\left(b^{2}+t\right)\left(c^{2}+t\right)\right\}^{\frac{1}{2}}} d t
$$

normalized such that $\phi(\infty)=0$, and valid for all relative values of $a, b, c$, (not just $a>b>c$ ).

\section{B. 2-D Gaussian charge distribution}

The potential for the 2-D Gaussian charge distribution can be obtained from (5.2) by proceeding to the limit $c \rightarrow \infty$. The charge per unit length, $\tau$, for finite $z$, is

$$
\tau=\frac{Q}{c \sqrt{ } \pi}
$$

in which case $(4.20)$ leads to

$$
\phi_{2 G}(x, y)=-\frac{\tau}{4 \pi \varepsilon_{0}} \int_{0}^{\infty} d t \frac{1-\exp \left(-\frac{x^{2}}{a^{2}+t}-\frac{y^{2}}{b^{2}+t}\right)}{\left\{\left(a^{2}+t\right)\left(b^{2}+t\right)\right\}^{\frac{1}{2}}}
$$

where the potential is normalized such that $\phi(0)=0$. With this normalization, it is clear that $\phi(\infty) \rightarrow-\infty$, as expected.

\section{3-D uniform distribution}

In this case we choose

$$
f(s)=\left\{\begin{array}{cc}
\frac{3 Q}{4 \pi a b c}, & s<1 \\
0, & s>1
\end{array}\right\}
$$

in which case

$$
\int_{s(t)}^{\infty} d s f(s)=\left\{\begin{array}{cc}
3 Q(7-s) /(4 \pi a b c) & , s<1 \\
0 & , s>1
\end{array}\right\}
$$


Thus (4.19) becomes

$$
\phi_{3 U}(x, y, z)=\frac{3 Q}{16 \pi \varepsilon_{0}}\left\{\begin{array}{ll}
\int_{0}^{\infty} d t \frac{\left(1-\frac{x^{2}}{a^{2}+t}-\frac{y^{2}}{b^{2}+t}-\frac{z^{2}}{c^{2}+t}\right)}{\left\{\left(a^{2}+t\right)\left(b^{2}+t\right)\left(c^{2}+t\right)\right\}^{\frac{1}{2}}}, \frac{x^{2}}{a^{2}}+\frac{y^{2}}{b^{2}}+\frac{z^{2}}{c^{2}}<1 \\
\int_{t_{0} d t}^{\infty} & , \frac{x^{2}}{a^{2}}+\frac{y^{2}}{b^{2}}+\frac{z^{2}}{c^{2}}>1
\end{array}\right\}
$$

where $t_{0}(x, y, z)$ is the value of $t_{0}$ for which

$$
\frac{x^{2}}{a^{2}+t_{0}}+\frac{y^{2}}{b^{2}+t_{0}}+\frac{z^{2}}{c^{2}+t_{0}}=1
$$

Inside the distribution, $\phi$ is a linear function of $x^{2}, y^{2}, z^{2}$ whose coefficients are elliptic integrals.

\section{2-D uniform distribution}

The potential for the 2-D uniform distribution is obtained once again by proceeding to the limit $c \rightarrow \infty$, in which case $Q=4 \tau c / 3$, so that $(4.20)$ leads to

$$
\phi_{2 U}(x, y)=\frac{-\tau}{4 \pi \varepsilon_{0}}\left\{\begin{array}{cc}
\int_{0}^{\infty} \frac{\left(\frac{x^{2}}{a^{2}+t}+\frac{y^{2}}{b^{2}+t}\right)}{\left\{\left(a^{2}+t\right)\left(b^{2}+t\right)\right\}^{\frac{1}{2}}} d t & \frac{x^{2}}{a^{2}}+\frac{y^{2}}{b^{2}}<1 \\
t_{0} d t & \frac{x^{2}}{a^{2}+t}+\frac{y^{2}}{b^{2}+t} \\
\int_{0}^{\left\{\left(a^{2}+t\right)\left(b^{2}+t\right)\right\}^{\frac{1}{2}}}+\int_{t_{0}}^{\left\{\left(a^{2}+t\right)(b 2+t)\right\}^{\frac{1}{2}} d t}, \\
x^{2} / a^{2}+y^{2} / b^{2}>1
\end{array}\right\}
$$

Here $t_{0}(x, y)$ is the value of $t_{0}$ for which

$$
x^{2} /\left(a^{2}+t_{0}\right)+y^{2} /\left(b^{2}+t_{0}\right)=1
$$


The potential is normalized such that $\phi(0)=0$, leading necessarily to $\phi(\infty) \rightarrow-\infty$. Within the ellipse, one can easily show that

$$
\phi_{2 U}(x, y)=\frac{-\tau}{2 \pi \varepsilon_{0}(a+b)}\left(\frac{x^{2}}{a}+\frac{y^{2}}{b}\right)
$$

leading to the usual linear space charge force for a Kapchinskij-Vladimirskij phase space distribution. ${ }^{2}$

\section{References}

1. J. A. Stratton, Electromagnetic Theory, McGraw-Hi11, Inc. (1941), pp. 58-59 \& pp. 207-209.

2 I. M. Kapchinskij and V. V. Vladimirskij, Conference on High Energy Accelerators and Instrumentation, CERN, Geneva (1959), p. 274. 